\title{
On the maximum rotational frequency of neutron and hybrid stars
}

\author{
G. F. Burgio ${ }^{1}$, H.-J. Schulze ${ }^{1}$, and F. Weber ${ }^{2}$ \\ 1 Istituto Nazionale di Fisica Nucleare, Sezione di Catania, Via S. Sofia 64, 95123 Catania, Italy \\ 2 Department of Physics, San Diego State University, 5500 Campanile Drive, San Diego, CA 92182, USA
}

Received 26 February 2003 / Accepted 18 June 2003

\begin{abstract}
We construct self-consistent equilibrium sequences of general relativistic, rotating neutron star models. Special emphasis in put on the determination of the maximum rotation frequency of such objects. Recently proposed models for the equation of state of neutron star matter are employed, which are derived by describing the hadronic phase within the many-body Brueckner-Bethe-Goldstone formalism, and the quark matter phase within the MIT bag model using a density dependent bag constant. We find that the rotational frequencies of neutron stars with deconfined quark phases in their cores rival those of absolutely stable, self-bound strange quark matter stars. This finding is of central importance for the interpretation of extremely rapidly rotating pulsars, which are the targets of present pulsar surveys.
\end{abstract}

Key words. dense matter - equation of state - stars: neutron - stars: rotations

\section{Introduction}

Bulk properties of neutron stars depend sensitively on the equation of state (EOS) of nuclear matter at supernuclear density (Shapiro \& Teukolsky 1983). In fact, matter in the cores of neutron stars possesses densities ranging from a few times $n_{0}$ $\left(\approx 0.17 \mathrm{fm}^{-3}\right.$, the normal nuclear matter density) up to one order of magnitude higher. The EOS at such extreme densities is only poorly known. In the simplest model, a neutron star consists of neutrons only. In a slightly more accurate representation, a neutron star will contain neutrons and a small number of protons whose charge is balanced by leptons. Because of the extreme densities in the interior of a neutron star, the neutron chemical potential will exceed the mass (modified by interactions) of various members of the baryon octet. So in addition to neutrons, protons, and electrons, neutron stars may be expected to have populations of hyperons, too (Glendenning 1982, 1985). A comprehensive description of neutron star matter should thus account not only for nucleons and leptons, but also for hyperons, mesons, and the plausible transition to a quark-gluon plasma state (Glendenning 1982; Baym et al. 1985; Glendenning 1985, 1990; Weber 1999a,b; Glendenning 2000).

Because of the unprecedented advances in observational astronomy, one may hope that measurements of neutron star parameters will soon constrain theories of dense matter. The rotational periods of rapidly rotating neutron stars (pulsars), for instance, provide restrictions on the EOS when combined with the mass constraint. Radio pulsars are stable rotators which can be treated as uniformly rotating bodies (Weber 1999a). Their rotational frequencies are bound from above by the Kepler

Send offprint requests to: $\mathrm{G}$. F. Burgio, e-mail: fiorella.burgio@ct.infn.it frequency $\Omega_{\mathrm{K}}$, which sets an absolute limit on stable rotation because of mass shedding from the star's equator. $\Omega_{\mathrm{K}}$ depends sensitively on the EOS (Friedman et al. 1986, 1989; Lattimer et al. 1990; Cook et al. 1994; Salgado et al. 1994; Weber 1999a,b). An EOS which predicts Kepler frequencies that are smaller than the observed rotational frequencies is to be rejected as it is not compatible with observation.

In this paper we present results for configurations that are rotating uniformly at their Kepler frequencies. Models for the EOS of dense neutron star matter are computed for three different descriptions which are based on the microscopic manybody Brueckner-Bethe-Goldstone (BBG) theory (Baldo 1999). The first EOS describes hadronic matter with nucleons and leptons in beta-equilibrium (Baldo et al. 1997). The second EOS includes the presence of all hyperons $\left(\Sigma^{-}\right.$and $\left.\Lambda\right)$ that become populated in neutron star matter up to the highest densities relevant for neutron stars (Baldo et al. 2000a). Finally, the third EOS accounts for a hypothetical phase transition of confined hadronic matter into deconfined quark matter (Burgio et al. 2002a,b). The hadronic phase is described in the framework of the BBG theory, and the deconfined quark phase within an extended MIT bag model (Chodos et al. 1974). We use recent experimental results on the possible formation of a quarkgluon plasma state (Heinz 2000) at CERN to constrain the bag constant, $B$, which turns out to be density dependent.

Calculations of neutron star (NS) properties performed for such an EOS show that the maximum NS masses lie in the relatively narrow interval of $1.4 M_{\odot} \leq M_{\max } \leq 1.7 M_{\odot}$. Moreover, we find that neutron stars with deconfined quark matter in their interiors computed for an effective density-dependent bag constant, can rotate at Kepler periods down to half a millisecond. Such small rotational periods are not accessible to neutron stars made of confined hadronic matter only nor to neutron stars with 
a hadron-quark phase transition computed for a constant bag constant. It was commonly believed that only absolutely stable strange stars (Witten 1984), which are self-bound objects, may rotate that rapidly (Glendenning 1990). Our findings, however, suggest that pulsars rotating with about half a millisecond period could still be interpreted as conventional neutron stars containing a metastable deconfined quark phase at their centers.

This paper is organized as follows. In Sect. 2 we briefly illustrate the BBG many-body theory. We then proceed to the discussion of the hadron-quark phase transition described within the MIT bag model supplemented with a density dependent bag constant. The stellar structure equations are introduced in Sect. 3. In Sect. 4 we present our results for both non-rotating as well as for rotating neutron stars and discuss the impact of an effective, density dependent bag constant on the Kepler frequency. Finally, conclusions are drawn in Sect. 5.

\section{Equations of state of neutron star matter}

We begin with the description of the hadronic phase, for which we have adopted the Brueckner-Bethe-Goldstone (BBG) theory. This microscopic many-body approach is based on a linked cluster expansion of the energy per nucleon of nuclear matter (Baldo 1999). The basic ingredient is the Brueckner reaction matrix $G$, which is the solution of the Bethe-Goldstone equation,

$G[n ; \omega]=V+\sum_{k_{a} k_{b}} V \frac{\left|k_{a} k_{b}\right\rangle Q\left\langle k_{a} k_{b}\right|}{\omega-e\left(k_{a}\right)-e\left(k_{b}\right)} G[n ; \omega]$,

where $V$ is the bare nucleon-nucleon (NN) interaction, $n$ is the nucleon number density, and $\omega$ the starting energy. The singleparticle energy $e(k)$ (assuming $\hbar=1)$,

$e(k)=e(k ; n)=\frac{k^{2}}{2 m}+U(k ; n)$,

and the Pauli operator $Q$ constrain the propagation of intermediate baryon pairs above the Fermi momentum. The Brueckner-Hartree-Fock (BHF) approximation for the singleparticle potential $U(k ; n)$ using the continuous choice is

$$
U(k ; n)=\operatorname{Re} \sum_{k^{\prime} \leq k_{\mathrm{F}}}\left\langle k k^{\prime}\left|G\left[n ; e(k)+e\left(k^{\prime}\right)\right]\right| k k^{\prime}\right\rangle_{a},
$$

where the subscript " $a$ " indicates anti-symmetrization of the matrix elements. Because of the occurrence of $U(k ; n)$ in Eq. (2), Eqs. (1) through (3) constitute a coupled system of equations that need to be solved self-consistently for different densities (i.e., Fermi momenta). The basic input quantity in the Bethe-Goldstone Eq. (1) is the NN interaction in free space, $V$. We adopt the elaborate Paris potential (Lacombe et al. 1980) as a model for the free two-nucleon interaction supplemented with the Urbana model UVII as three-body force (Carlson et al. 1983; Schiavilla et al. 1986). In the BHF approximation the energy per nucleon is given by

$$
\frac{E}{A}=\frac{3}{5} \frac{k_{\mathrm{F}}^{2}}{2 m}+\frac{1}{2 n} \operatorname{Re} \sum_{k, k^{\prime} \leq k_{\mathrm{F}}}\left\langle k k^{\prime}\left|G\left[n ; e(k)+e\left(k^{\prime}\right)\right]\right| k k^{\prime}\right\rangle_{a} .
$$

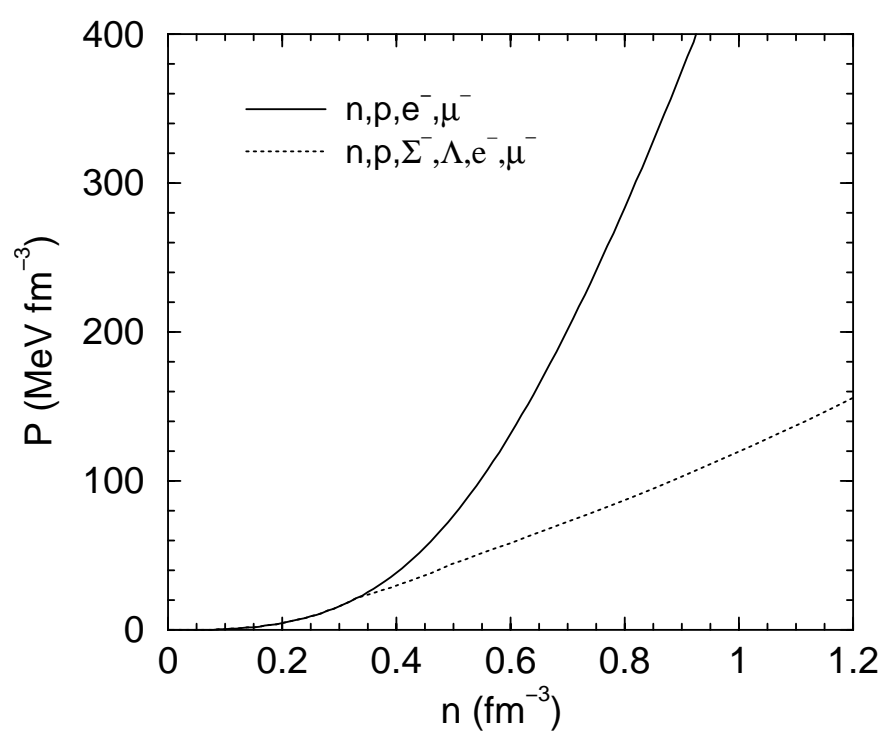

Fig. 1. BBG equation of state (EOS) of asymmetric, electrically charge neutral hadronic matter in beta equilibrium. The solid line shows the EOS when only nucleons and leptons are present, whereas the dotted line shows the EOS when hyperons are included.

Higher order correlations have been demonstrated to be negligible over the considered density range (Baldo et al. 2000b, 2001). The nuclear matter EOS computed from Eqs. (1)(4) fulfills several essential requirements, namely (i) it reproduces the correct nuclear matter saturation density, i.e., $n_{0}=$ $0.176 \mathrm{fm}^{-3}$, (ii) the nuclear incompressibility $K=281 \mathrm{MeV}$ is compatible with the values extracted from phenomenology, (iii) the symmetry energy $a_{\text {sym }}=33.6 \mathrm{MeV}$ is compatible with nuclear phenomenology, and (iv) the causality condition is strictly fulfilled (Baldo et al. 1997).

Recently, we incorporated hyperons into the BBG treatment of neutron star matter (Baldo et al. 1998, 2000a). This requires knowledge of the nucleon-hyperon $(\mathrm{NH})$ and hyperonhyperon $(\mathrm{HH})$ interactions. Because of the lack of experimental data, the hyperon-hyperon interaction has been neglected in a first approximation, whereas for the NH interaction the Nijmegen soft-core model (Maessen et al. 1989) has been adopted.

The equation of state of neutron star matter calculated along this scheme is shown in Fig. 1. The solid line displays the pressure as a function of baryon number density of beta-stable, electrically charge neutral nuclear matter when only nucleons and leptons are present. The dashed line represents the equation of state when $\Sigma^{-}$and $\Lambda$ hyperons are self-consistently included, too. It is evident that the presence of hyperons strongly softens the equation of state (Glendenning 1982, 1985). This is caused by both the larger number of baryonic degrees of freedom and the attraction felt by the $\Sigma^{-}$in the nuclear medium. As it turns out, this model for the EOS produces a maximum neutron star mass that lies slightly below the canonical value of $1.44 M_{\odot}$ (Taylor \& Weisberg 1989).

We now turn to the description of the deconfined quark phase. To describe this phase, we have used the MIT bag model (Chodos et al. 1974). In this model, the total energy density is the sum of a non-perturbative energy shift $B$, the bag constant, 

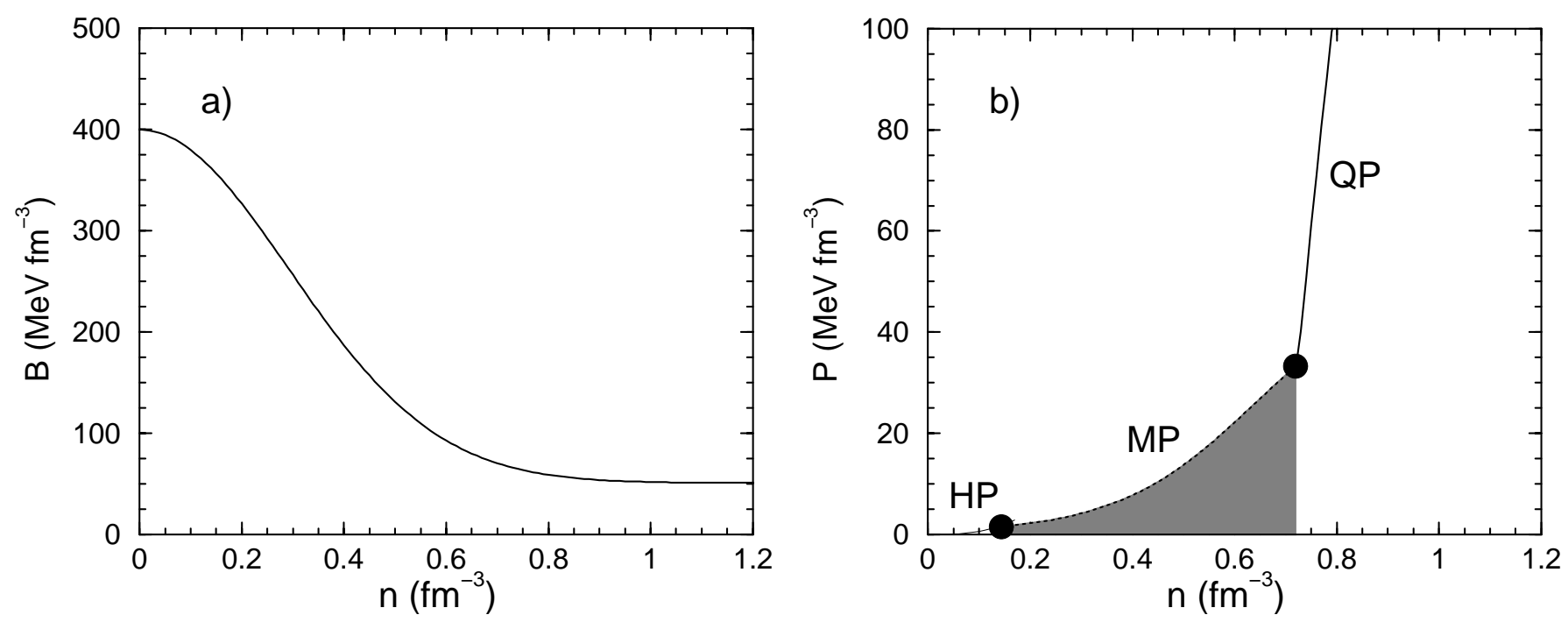

Fig. 2. Bag constant $B$ versus baryon number density for a Gaussian parametrization (panel a)). Calculations are performed for a value of the QCD coupling constant of $\alpha_{s}=0$. In panel b) the total EOS including both hadronic and quark components is displayed. The shaded region, bordered by two dots, indicates the mixed phase (MP) of quarks and hadrons, while HP and QP label the portions of the EOS where pure hadron or pure quark phases are present.

and the kinetic energy for non-interacting massive quarks of flavors $f$ with masses $m_{f}$ and Fermi momenta $k_{\mathrm{F}}^{(f)}\left[=\left(\pi^{2} n_{f}\right)^{1 / 3}\right.$, where $n_{f}$ denotes the density of quark flavor $\left.f\right]$ :

$\frac{E}{V}=B+\sum_{f} \frac{3 m_{f}^{4}}{8 \pi^{2}}\left[x_{f}\left(2 x_{f}^{2}+1\right) \sqrt{x_{f}^{2}+1}-\operatorname{arsinh} x_{f}\right]$

with $x_{f}=k_{\mathrm{F}}^{(f)} / m_{f}$. The $u$ and $d$ quarks are considered to be massless, whereas the $s$ quark mass is chosen to be $150 \mathrm{MeV}$. In the original MIT bag model, the bag constant $B$ has a value of $B \approx 55 \mathrm{MeV} \mathrm{fm}^{-3}$, which is quite small when compared with those $\left(\approx 210 \mathrm{MeV} \mathrm{fm}^{-3}\right)$ estimated from lattice calculations (Satz 1982). In this sense $B$ can be considered as a free parameter of the model.

In an earlier work, Burgio et al. (2002a) have proposed a method to constrain $B$ by experimental data obtained at the CERN SPS, where several experiments using high energy beams of $\mathrm{Pb}$ nuclei reported (indirect) evidence for the formation of a quark-gluon plasma (Heinz 2000). According to the analysis of those experiments, the quark-hadron transition takes place at about seven times normal nuclear matter energy density $\left(\epsilon_{0} \approx 156 \mathrm{MeV} \mathrm{fm}^{-3}\right)$. By assuming that the transition to the quark-gluon plasma is determined by the value of the energy density only, Burgio et al. (2002a) found that a density dependent bag parameter is needed in order to reproduce correctly the experimentally found value of the energy density at the hadron-quark transition point. More specifically, a Gaussianlike or a Woods-Saxon-like density dependence of $B$ has been assumed, and parameters were fitted in order to correctly reproduce the experimental phase transition point. More details are given in (Burgio et al. 2002a,b). We display in Fig. 2a the bag constant $B$ as function of the baryon density $n$ in the case that the assumed parametrization is a Gaussian.

Once the density dependence of $B$ is established, the composition of electrically charge neutral, $\beta$-stable quark matter can be determined. The density range over which both hadronic matter and quark matter coexist is computed as outlined in (Glendenning 1992) for a system characterized by two conserved charges. The hadronic phase and the quark phase are allowed to be separately electrically charged while, at the same time, total charge neutrality is preserved. The pressure in the two phases is the same to ensure mechanical stability, while the chemical potentials of the different species are related to each other through baryon number conservation and electric charge conservation.

The resulting EOS for neutron star matter, for the Gaussianlike bag parametrization, is displayed in Fig. $2 b$, where the shaded area indicates the mixed phase region. A pure quark phase is present at densities above the shaded area and a pure hadronic phase is present below this area. Moreover, the onset density of the mixed phase turns out to be slightly smaller than the density for hyperon formation in pure hadronic matter. Of course hyperons are still present in the hadronic component of the mixed phase.

\section{Stellar structure equations of rotating stars}

The construction of general relativistic rotating neutron star models is a complicated task (see, for instance, Butterworth \& Ipser 1976; Friedman et al. 1986, 1989; Komatsu et al. 1989; Lattimer et al. 1990; Salgado et al. 1994). We adopt Hartle's method (Hartle 1967; Hartle \& Thorne 1968) to achieve this goal. The metric has the form

$\mathrm{d} s^{2}=-\mathrm{e}^{2 v} \mathrm{~d} t^{2}+\mathrm{e}^{2 \psi}(\mathrm{d} \phi-\omega \mathrm{d} t)^{2}+\mathrm{e}^{2 \mu} \mathrm{d} \theta^{2}+\mathrm{e}^{2 \lambda} \mathrm{d} r^{2}+O\left(\Omega^{3}\right),(6)$

where $v, \psi, \mu, \lambda$ denote metric functions and $\omega$ is the angular velocity of a local inertial frame. The general relativistic Kepler frequency, denoted by $\Omega_{\mathrm{K}}$, is given as a solution of (Friedman et al. 1986; Weber 1999a,b)

$\Omega=\mathrm{e}^{\nu-\psi} v(\Omega)+\omega(\Omega)$, 

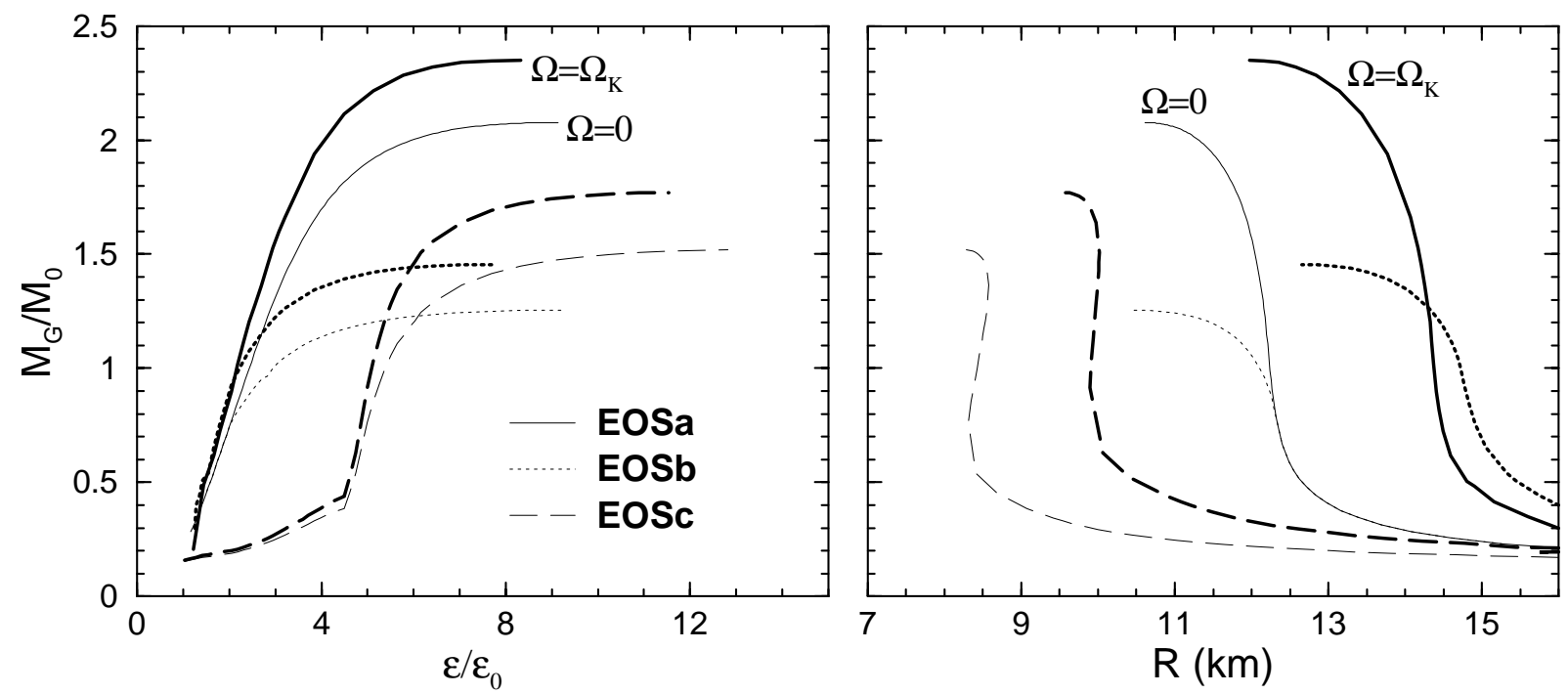

Fig. 3. The gravitational mass (in units of the mass of the Sun, $M_{\odot}$ ) versus the normalized central energy density (left panel) and versus the equatorial radius (right panel). The thin lines represent static equilibrium configurations, whereas the thick lines display configurations rotating at their respective Kepler frequencies. Several different stellar matter compositions are considered (see text for details).

$v(\Omega)=\frac{\omega^{\prime}}{2 \psi^{\prime}} \mathrm{e}^{\psi-v}+\sqrt{\frac{v^{\prime}}{\psi^{\prime}}+\left(\frac{\omega^{\prime}}{2 \psi^{\prime}} \mathrm{e}^{\psi-\nu}\right)^{2}}$,

where $v$ denotes the orbital velocity at the star's equator. Primes refer to derivatives with respect to the radial coordinate. All quantities in Eqs. (7) and (8) are to be evaluated at the star's equator. Equations (6) to (8) are to be solved self-consistently for a given model for the EOS. Details can be found in Weber (1999a).

\section{Results and discussion}

In Fig. 3 we plot our results obtained for non-rotating neutron stars (thin lines) as well as for neutron stars rotating at their self-consistent Kepler frequencies (thick lines). The vertical axes display the gravitational mass (in units of solar masses) as a function of central star density, $\epsilon_{\mathrm{c}}$, normalized to the saturation value of infinite nuclear matter, $\epsilon_{0}=156 \mathrm{MeV} \mathrm{fm}^{-3}$. On the right-hand side in Fig. 3 we illustrate the gravitational mass as function of the equatorial radius. The solid curves show results for neutron star matter composed of only nucleons and leptons (EOSa). The dotted lines represent equilibrium configurations of neutron stars composed of nucleons, hyperons, and leptons. The dashed curves display equilibrium configurations of hybrid stars, i.e., neutron stars composed of hadronic matter in the outer layers, followed by a mixed phase of quarks and hadrons at higher densities, and eventually a pure quark matter core in their centers (EOSc).

Our results for the gravitational mass, normalized central density, equatorial radius, and Kepler frequency for the maximum-mass star of each stellar sequence are displayed in Table 1. One sees an enhancement of the gravitational mass for rotating configurations, relative to the spherical star model of the same central energy density. This enhancement lies in
Table 1. Calculated properties of non-rotating $(\Omega=0)$ and rotating $\left(\Omega=\Omega_{\mathrm{K}}\right)$ limiting-mass neutron star models.

\begin{tabular}{lcccc}
\hline \hline EOS & $\Omega\left(10^{4} \mathrm{~s}^{-1}\right)$ & $M_{\mathrm{G}} / M_{\odot}$ & $\epsilon_{\mathrm{c}} / \epsilon_{0}$ & $R_{\mathrm{eq}}(\mathrm{km})$ \\
\hline EOSa & 1.195 & 2.35 & 8.33 & 11.97 \\
& 0 & 2.06 & 8.97 & 10.50 \\
EOSb & 0.88 & 1.45 & 7.7 & 12.60 \\
& 0 & 1.26 & 8.97 & 10.46 \\
EOSc & 1.436 & 1.77 & 11.54 & 9.60 \\
& 0 & 1.52 & 12.8 & 8.28 \\
\hline
\end{tabular}

the range between $\approx 14 \%$ and $17 \%$, depending on the underlying EOS. Correspondingly, for the same value of the gravitational mass, the rotating configurations exhibit reduced central densities compared to the corresponding non-rotating stars, which is due to the effect of the centrifugal force, which effectively stiffens the equation of state. Moreover, the mass increase is accompanied by a relatively large increase of the equatorial radius, due to the rotational deformation of the star.

A striking difference between the purely hadronic stars (EOSa and EOSb) and the hybrid stars (EOSc) concerns their compactness. In fact, for a fixed gravitational mass, hybrid stars are characterized by a larger central energy density and a smaller radius than their hadronic counterparts. This holds for both static as well as rotating configurations, as one would expect. The compactness of a star is crucial for its Kepler frequency as discussed next.

In Fig. 4 we display the Kepler periods $P_{\mathrm{K}}\left(=2 \pi / \Omega_{\mathrm{K}}\right)$ in milliseconds versus the rotational star mass for several different stellar sequences based on different EOSs. The shaded area represents the current range of the observed periods and masses.

Since the observed periods are larger than the Kepler values, which set an absolute limit on stable rapid rotation, all 


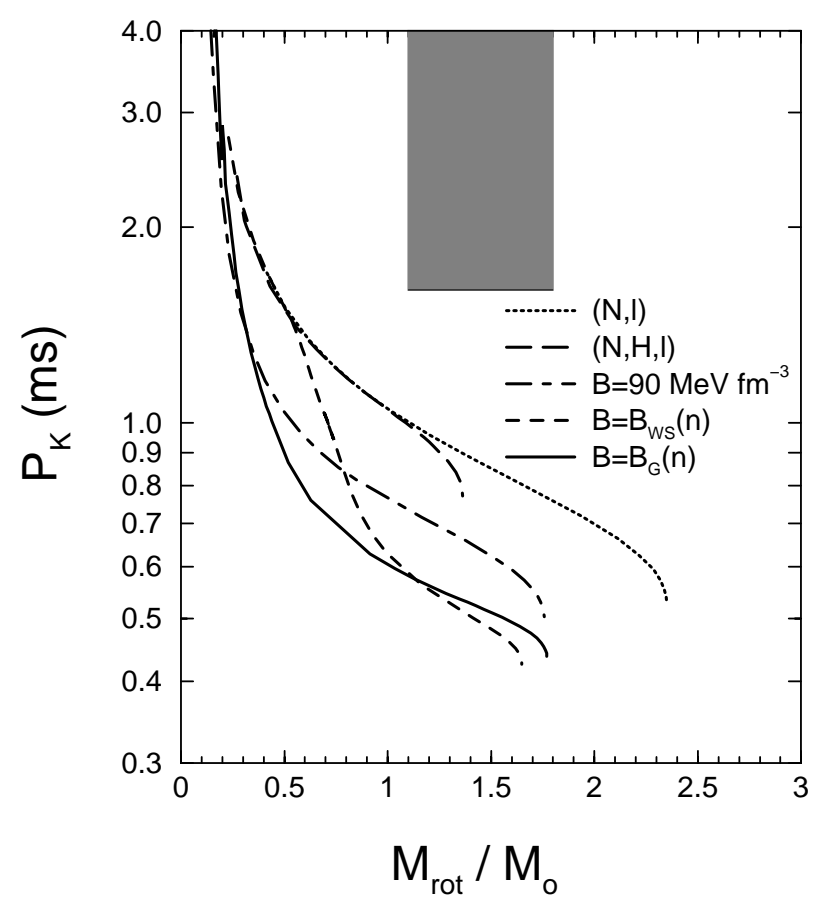

Fig. 4. Kepler period versus the rotational mass for purely hadronic stars as well as hybrid stars. The following core compositions are considered: i) nucleons and leptons (dotted line), ii) nucleons, hyperons, and leptons (long-dashed line), iii) hadrons and quarks [solid line: $B=$ $B_{\mathrm{G}}(n)$, dot-dashed line: $B=90 \mathrm{MeV} \mathrm{fm}^{-3}$, dashed line: $\left.B=B_{\mathrm{WS}}(n)\right]$. The shaded area represents the current range of observed data.

EOSs studied in this paper are compatible with the observed data. Stars made up of chemically equilibrated nucleons or nucleons plus hyperons, shown by the dotted and long-dashed lines respectively, show instability against mass shedding first because of their relatively large equatorial radii. In both cases, the limiting mass configurations are characterized by values of the Kepler period larger than half a millisecond, in agreement with results usually found in the literature (Weber 1999a,b). This is different for the Kepler periods computed for the hybrid stars in Fig. 4 (labeled $B=B_{\mathrm{G}}(n), B=90 \mathrm{MeV} \mathrm{fm}^{-3}$, and $\left.B=B_{\mathrm{WS}}(n)\right)$ which can be even smaller than half a millisecond. An example are the configurations along the solid line near the mass limit, which have been obtained using a Gaussian parametrization of the bag constant. We stress that this result does not depend on the particular parametrization chosen for the bag constant $B$. In fact, stellar configurations obtained with a Woods-Saxon-like bag parametrization (dashed line) show the same behavior.

For completeness, we show in Fig. 4 also the Kepler periods of hybrid stars computed for a quark matter EOS with a constant bag constant of $B=90 \mathrm{MeV} \mathrm{fm}^{-3}$. In this case, no equilibrium configuration can rotate at Kepler periods smaller than half a millisecond. This is due to the fact that the increase of pressure with baryon density in the pure quark phase is less pronounced than in the case of a density dependent bag value, as shown in (Burgio et al. 2002a). Therefore, we deduce that only hybrid stars with a quark phase constructed by using a density-dependent bag constant can rotate stably at periods in the half millisecond regime. The Kepler periods of such hybrid stars therefore rival those of absolutely stable strange stars (Glendenning 1990), which were thought to be the only configurations able to withstand stable rotation down to half millisecond periods.

\section{Summary}

In this work, we have explored the Kepler periods of sequences of general relativistic, rotating neutron and hybrid stars. For this purpose we have employed three different equations of state derived by describing the hadronic phase in the framework of the many-body Brueckner-Bethe-Goldstone theory using state-of-the-art nucleon-nucleon interactions, and the quark phase within the MIT bag model supplemented by a density dependent bag constant $B$, as suggested by experiment. In essence, we have found that neutron stars made of purely hadronic matter (nucleons and hyperons) have minimum rotational periods that are clearly larger than half a millisecond. This may be different for hybrid stars, i.e., neutron stars containing deconfined quark matter cores. In the latter case, a density dependent bag constant provides additional compactness to hybrid stars so that Kepler periods down to half a millisecond are reached. Such small rotational periods are not obtained if the bag parameter is taken to be constant, as usually assumed in the studies published in the literature.

We thus conclude that very rapidly rotating pulsars, which are being actively searched for by upgraded radio telescopes and the latest generation of X-ray satellites, may be interpreted as either strange quark matter stars or neutron stars with deconfined quark matter interiors.

Acknowledgements. We warmly thank Marcello Baldo (INFN Sezione di Catania) for the fruitful discussions about the hadron-quark phase transition in neutron stars.

\section{References}

Baldo, M. 1999, Nuclear Methods and the Nuclear Equation of State (Singapore: World Scientific)

Baldo, M., Bombaci, I., \& Burgio, G. F. 1997, A\&A, 328, 274

Baldo, M., Burgio, G. F., \& Schulze, H.-J. 1998, Phys. Rev. C, 58, 3688

Baldo, M., Burgio, G. F., \& Schulze, H.-J. 2000a, Phys. Rev. C, 61, 055801

Baldo, M., Song, H. Q., Giansiracusa, G., \& Lombardo, U. 2000b, Phys. Lett. B, 473, 1

Baldo, M., Fiasconaro, A., Song, H. Q., Giansiracusa, G., \& Lombardo, U. 2001, Phys. Rev. C, 65, 017303

Baym, G., Kolb, E. W., McLerran, L., Walker, T. P., \& Jaffe, R. L. 1985, Phys. Lett. B, 160, 181

Burgio, G. F., Baldo, M., Sahu, P. K., Santra, A. B., \& Schulze, H.-J. 2002a, Phys. Lett. B, 526, 19

Burgio, G. F., Baldo, M., Sahu, P. K., \& Schulze, H.-J. 2002b, Phys. Rev. C, 66, 025802

Butterworth, E. M., \& Ipser, J. R. 1976, ApJ, 204, 200

Carlson, J., Pandharipande, V. R., \& Wiringa, R. B. 1983, Nucl. Phys. A, 401,59 
Chodos, A., Jaffe, R. L., Johnson, K., Thorn, C. B., \& Weisskopf, V. F. 1974, Phys. Rev. D, 9, 3471

Cook, G. B., Shapiro, S. L., \& Teukolsky, S. A. 1994, ApJ, 424, 823

Friedman, J. L., Ipser, J. R., \& Parker, L. 1986, ApJ, 304, 115

Friedman, J. L., Ipser, J. R., \& Parker, L. 1989, Phys. Rev. Lett., 62, 3015

Glendenning, N. K. 1982, Phys. Lett. B, 114, 391

Glendenning, N. K. 1985, ApJ, 293, 470

Glendenning, N. K. 1990, Mod. Phys. Lett. A, 5, 2197

Glendenning, N. K. 1992, Phys. Rev. D, 46, 1274

Glendenning, N. K. 2000, Compact Stars, Nuclear Physics, Particle Physics, and General Relativity, 2nd ed. (New York: Springer-Verlag)

Hartle, J. 1967, ApJ, 150, 1005

Heinz, U., \& Jacobs, M. [nucl-th/0002042]

Heinz, U. [hep-ph/0009170]

Komatsu, H., Eriguchi, Y., \& Hachisu, I. 1989, MNRAS, 237, 355; 239,153
Lacombe, M., Loiseau, B., Richard, J. M., et al. 1980, Phys. Rev. C, 21,861

Lattimer, J. M., Prakash, M., Masak, D., \& Yahil, A. 1990, ApJ, 355, 241

Maessen, P., Rijken, Th., \& de Swart, J. 1989, Phys. Rev. C, 40, 2226

Salgado, M., Bonazzola, S., Gourgoulhon, E., \& Haensel, P. 1994, A\&A, 291, 155; A\&AS, 108, 455

Satz, H. 1982, Phys. Rep., 89, 349

Schiavilla, R., Pandharipande, V. R., \& Wiringa, R. B. 1986, Nucl. Phys. A, 449, 219

Shapiro, S. L., \& Teukolsky, S. A. 1983, Black Holes, White Dwarfs, and Neutron Stars (New York: John Wiley \& Sons)

Taylor, J. H., \& Weisberg, J. M. 1989, ApJ, 345, 434

Weber, F. 1999a, Pulsars as Astrophysical Laboratories for Nuclear and Particle Physics (Bristol and Philadelphia: Institute of Physics Publishing)

Weber, F. 1999b, J. Phys. G: Nucl. Part. Phys., 25, R195

Witten, E. 1984, Phys. Rev. D, 30, 272 